\title{
Is age an additional factor in the treatment of elderly patients with glioblastoma? A new stratification model: an Italian Multicenter Study
}

\author{
Tamara lus, MD, PhD, ${ }^{1}$ Teresa Somma, MD, ${ }^{2}$ Roberto Altieri, MD, ${ }^{3}$ \\ Filippo Flavio Angileri, MD, PhD, ${ }^{5}$ Giuseppe Maria Barbagallo, MD,, 3 Paolo Cappabianca, MD, ${ }^{2}$ \\ Francesco Certo, MD, ${ }^{3,4}$ Fabio Cofano, MD, ${ }^{6}$ Alessandro D'Elia, MD, ${ }^{7}$ \\ Giuseppe Maria Della Pepa, MD, ${ }^{8}$ Vincenzo Esposito, MD, ${ }^{7,9}$ Marco Maria Fontanella, MD, ${ }^{10}$ \\ Antonino Germanò, MD, ${ }^{5}$ Diego Garbossa, MD, PhD, ${ }^{6}$ Miriam Isola, PhD, ${ }^{11}$ \\ Giuseppe La Rocca, MD, ${ }^{8,13}$ Francesco Maiuri, MD, ${ }^{2}$ Alessandro Olivi, MD, ${ }^{8}$ \\ Pier Paolo Panciani, MD, PhD, ${ }^{10}$ Fabrizio Pignotti, MD, ${ }^{13}$ Miran Skrap, MD, ${ }^{1}$ \\ Giannantonio Spena, MD,12 and Giovanni Sabatino, MD, PhD 8,13
}

\begin{abstract}
${ }^{1}$ Neurosurgery Unit, Department of Neurosciences, Santa Maria della Misericordia University Hospital, Udine; ${ }^{2}$ Division of Neurosurgery, Department of Neurosciences, Reproductive and Odontostomatological Sciences, Università degli Studi di Napoli Federico II, Naples; ${ }^{3}$ Department of Medical and Surgical Sciences and Advanced Technologies (G.F. Ingrassia); Neurological Surgery, Policlinico "G. Rodolico - San Marco" University Hospital, University of Catania; ${ }^{4}$ Interdisciplinary Research Center on Brain Tumors Diagnosis and Treatment, University of Catania; ${ }^{5}$ Division of Neurosurgery, BIOMORF Department, University of Messina; ${ }^{6}$ Department of Neuroscience "Rita Levi Montalcini," Neurosurgery Unit, University of Turin; ${ }^{7}$ Department of Neurosurgery "Giampaolo Cantore"-IRCSS Neuromed, Pozzilli; ${ }^{8}$ Institute of Neurosurgery, Fondazione Policlinico Gemelli, Catholic University, Rome; ' ${ }^{2}$ epartment of Human Neurosciences-"Sapienza" University of Rome; ${ }^{10}$ Division of Neurosurgery, Department of Surgical Specialties, Radiological Sciences and Public Health, University of Brescia; ${ }^{11}$ Department of Medicine, University of Udine; ${ }^{12}$ Department of Neurosurgery, Alessandro Manzoni Hospital, Lecco; and ${ }^{13}$ Department of Neurosurgery, Mater Olbia Hospital, Olbia, Italy
\end{abstract}

OBJECTIVE Approximately half of glioblastoma (GBM) cases develop in geriatric patients, and this trend is destined to increase with the aging of the population. The optimal strategy for management of GBM in elderly patients remains controversial. The aim of this study was to assess the role of surgery in the elderly ( $\geq 65$ years old) based on clinical, molecular, and imaging data routinely available in neurosurgical departments and to assess a prognostic survival score that could be helpful in stratifying the prognosis for elderly GBM patients.

METHODS Clinical, radiological, surgical, and molecular data were retrospectively analyzed in 322 patients with GBM from 9 neurosurgical centers. Univariate and multivariate analyses were performed to identify predictors of survival. A random forest approach (classification and regression tree [CART] analysis) was utilized to create the prognostic survival score.

RESULTS Survival analysis showed that overall survival (OS) was influenced by age as a continuous variable $(p=$ $0.018), M G M T(p=0.012)$, extent of resection (EOR; $p=0.002$ ), and preoperative tumor growth pattern (evaluated with the preoperative $T 1 / T 2 M R I$ index; $p=0.002$ ). CART analysis was used to create the prognostic survival score, forming six different survival groups on the basis of tumor volumetric, surgical, and molecular features. Terminal nodes with similar hazard ratios were grouped together to form a final diagram composed of five classes with different OSs $(p<0.0001)$. EOR was the most robust influencing factor in the algorithm hierarchy, while age appeared at the third node of the CART algorithm. The ability of the prognostic survival score to predict death was determined by a Harrell's C-index of $0.75(95 \%$ Cl 0.76-0.81).

CONCLUSIONS The CART algorithm provided a promising, thorough, and new clinical prognostic survival score for elderly surgical patients with GBM. The prognostic survival score can be useful to stratify survival risk in elderly GBM

ABBREVIATIONS CART = classification and regression tree; $\mathrm{CCI}=$ Charlson Comorbidity Index; EGBM = elderly GBM; EOR = extent of resection; GBM = glioblastoma; $\mathrm{HR}$ = hazard ratio; KPS = Karnofsky Performance Scale; OS = overall survival; PFS = progression-free survival; RHR = relative HR.

SUBMITTED May 25, 2020. ACCEPTED July 23, 2020.

INCLUDE WHEN CITING DOI: 10.3171/2020.7.FOCUS20420. 
patients with different surgical, radiological, and molecular profiles, thus assisting physicians in daily clinical management. The preliminary model, however, requires validation with future prospective investigations. Practical recommendations for clinicians/surgeons would strengthen the quality of the study; e.g., surgery can be considered as a first therapeutic option in the workflow of elderly patients with GBM, especially when the preoperative estimated EOR is greater than $80 \%$.

https://thejns.org/doi/abs/10.3171/2020.7.FOCUS20420

KEYWORDS glioblastoma surgery; elderly; prognostic score; extent of resection; CART model; classification and regression tree; decision tree diagram

$\mathrm{T}$ HE prognosis of glioblastoma (GBM) is universally poor, especially in elderly patients, in whom the median survival ranges from 4 to 9 months. ${ }^{1-9}$ Approximately half of GBM cases occur in geriatric patients, and this trend is destined to increase with the aging of the population.

The optimal strategy for management of GBM in elderly patients (EGBM) remains controversial, especially in regard to the effects of extent of resection (EOR) on survival outcomes. ${ }^{10-19}$ There is overwhelming evidence to suggest that survival and neurological function ${ }^{4}$ outcomes can be optimized through maximal safe resection in younger patients with GBM. However, many neurosurgeons tend to avoid aggressive surgical interventions in EGBM patients because of the probable increased risk of perioperative complications..$^{1,17-19}$ Life expectancy, overall health status, and quality of life in the elderly, however, are all increasing globally, which makes a strong case for redefining the concept of "elderly" and reframing it in the context of GBM surgical management.

Considering that the incidence of GBM is higher within this expanding age group of the older population, it is of utmost importance to identify prognostic factors and effective therapeutic strategies for improving survival and quality of life. ${ }^{17,18}$ An increasing number of prognostic survival tools are being developed to combine clinical, radiological, and molecular variables in an all-inclusive risk stratification model. ${ }^{20-22}$ Given the importance of each individual factor, it is often difficult to establish how these interact with each other and how they impact prognosis in the complexity of clinical settings. Cox survival analysis generally detects risk factors without highlighting how their interactions or various combinations influence the prognosis. The algorithms and computational statistics have already demonstrated an excellent performance in outcome predictions for a wide range of conditions, thus paving the way for a personalized medicine model. ${ }^{23}$

In light of this evidence, a multiparametric model for prognosis was elaborated, inclusive of radiological, molecular, and surgical variables, to assess prognosis in postoperative EGBM cases prior to postoperative treatment.

\section{Methods}

The methods of this study were based on a previous study in which a scoring system for patients of all ages with GBM was elaborated..$^{21}$

\section{Study Population and Inclusion Criteria}

A shared cooperative retrospective database of 322 adult patients surgically treated for newly diagnosed GBM between January 2015 and December 2018 was created. There is no generally agreed upon criterion for the definition of "older people." 17 To provide results that can be widely applied across countries, we used an age cutoff of 65 years old for defining older patients in the current research.

Patients were enrolled according to the following criteria: 1 ) age $\geq 65$ years; 2) no previous surgery; 3 ) no preoperative chemo- or radiotherapy; 4) presurgical evaluation using the Charlson Comorbidity Index (CCI); ${ }^{24}$ 5) objective evaluation of preoperative tumor volume on MR images in DICOM format based on postcontrast T1- and T2-weighted MRI sequences; 6) objective estimation of EOR on postcontrast T1-weighted MRI sequences; 7) revision of histopathological specimens using the new 2016 WHO classification of tumors of the CNS; and 8) MGMT promoter methylation and IDHI/IDH2 mutation status assessment. Exclusion criteria included needle biopsy, incomplete imaging data, follow-up interval, and multifocal tumors.

\section{Volumetric Analysis}

The neuroradiological tumor growth pattern, expressed by the preoperative T1/T2 MRI index, and EOR were computed as previously described. Briefly, the achieved EOR in each case was objectively evaluated using pre- and postoperative MR images (DICOM format), based on the contrast area of postcontrast T1-weighted MRI sequences, using the following formula: (preoperative tumor volume - postoperative tumor volume)/preoperative tumor volume. With the aim of evaluating the role of tumor growth pattern on overall survival (OS), the preoperative MRI index was assessed as follows: $\mathrm{T} 1 / \mathrm{T} 2$ = preoperative volumetric tumor volume on postcontrast T1-weighted images/ preoperative volumetric tumor volume on T2-weighted images. ${ }^{21,25}$

\section{Statistical Analysis}

Categorical variables were reported as percentages and continuous variables were reported as means \pm standard deviations or medians and ranges as appropriate, according to the data distribution. Normality of the continuous variables was tested using the Shapiro-Wilk test.

OS and progression-free survival (PFS) were estimated using the Kaplan-Meier approach. The association between variables and survival distribution was tested using univariate and multivariate Cox proportional hazard models (after verification of proportional hazard assumptions). Patients with unknown survival were censored as of their last scan date. The variables we considered for univariate analysis were age, sex, Karnofsky Performance Scale 
(KPS) score, preoperative tumor volume computed on postcontrast T1- and T2-weighted MR images, tumor location, tumor side, EOR, postoperative adjuvant protocol used, IDH1/2 mutation, MGMT methylation status, and $\mathrm{Ki}-67$. EOR was modeled as both a continuous and an ordinal variable $(\leq 79 \%, 80 \%-89 \%, 90 \%-99 \%$, and $100 \%)$ in univariate analysis to ensure consistency with previous studies that focused on the impact of glioma resection in terms of volumes.

In the univariate Cox regression, the preoperative $\mathrm{T} 1 /$ T2 MRI index was initially analyzed as a continuous variable. To better understand the variable's association pattern, the Cox regression model was then applied to the quintiles for this variable. Subsequently, the variable was dichotomized using a cutoff we identified at the quintile that showed a significant hazard ratio (HR). The variables that were significantly associated in the univariate model $(p<0.05)$ were included in the multivariate regression model, according to the stepwise-backward selection method. All statistical analyses were performed by Stata/ IC (version 13.0, StataCorp LP).

\section{Classification and Regression Tree Method}

To determine subgroup patients with different clinical prognoses, we used the decision tree model with the classification and regression tree (CART) method. ${ }^{21,26}$ This method is a machine learning model composed of hierarchical decision rules involving optimal cutoff values that recursively split independent factors into different groups. The groups of individuals are called nodes and form a branch node tree. Terminal nodes are groups of individuals that cannot be further subdivided on the basis of the established parameters (minimum size of subgroup, minimum number of events, and maximum $\mathrm{p}$ value required) to proceed in further subdivisions. In our study, nodes were required to have a minimum size of 20 patients, a minimum of 10 events, and a maximum $\mathrm{p}$ value of 0.05 . The significant variables in the univariate analysis were considered to generate the model. Once the regression tree was generated, the nodes of the terminal branches were pruned (aggregated) on the basis of their relative HRs (RHRs) to obtain final groups with homogeneous mortality risk. The final groups were converted into a prognostic survival score ordered according to their RHRs.

Differences in terms of OS probability among the score categories were investigated using univariate Cox regression analysis. The performance of the prognostic survival score in predicting time to death was estimated using Harrell's c-index. ${ }^{27}$ All statistical analyses were performed by Stata/IC (version 13.0, StataCorp LP).

\section{Results}

\section{Survival Risk Factors}

Table 1 lists the various features of the EGBM patients included in the study. The 1- and 2-year OS and PFS rates for the cohort were $42.07 \%$ and $14.89 \%$ (OS rates) and $24.8 \%$ and $9.64 \%$ (PFS rates), respectively. Univariate analysis indicated significantly improved OS in EGBM cases with the following features: young age $(p=0.035)$, high EOR ( $\mathrm{p}<0.0001)$, methylation of the MGMT promoter $(\mathrm{p}=0.002)$, presence of low residual tumor $(\mathrm{p}<$
0.0001), no corpus callosum involvement (0.040), and low preoperative T1/T2 MRI index ( $\mathrm{p}<0.0001)$. In the final model, variables with significant univariate analysis $\mathrm{p}$ values were included. Age ( $p=0.018)$, tumor involvement of the corpus callosum $(\mathrm{p}=0.023)$, preoperative T1/T2 MRI index $(\mathrm{p}=0.002)$, EOR $(\mathrm{p}=0.002)$, and MGMT methylation status ( $\mathrm{p}=0.012$ ) were found to be independent survival risk factors (Table 2).

\section{CART Model}

The CART analysis was applied to elaborate a promising, thorough, and new clinical prognostic survival score for EGBM patients based on surgical, neuroradiological, and molecular determinants. Specifically, the model generation is based on 3 phases of analysis as reported in our previous study: ${ }^{21}$ 1) the Kaplan-Meier approach was used to identify the most important survival factors; 2) a decision tree algorithm was applied to stratify OS in different prognosis groups; and 3) the prognostic survival score was computed. In detail, the CART model derives from independent predictor factors detected by the univariate analysis (age, preoperative tumor T1/T2 MRI index, tumor involvement of corpus callosum on preoperative MRI, EOR, MGMT methylation status, residual tumor evidenced on postcontrast T1-weighted MR images, and IDHI/2 mutation status).

First, the CART analysis was performed on 250 cases that met all the selection criteria, leading to the definition of 6 terminal nodes. Terminal nodes with similar RHRs were grouped together to form a final diagram composed of 5 classes with different OSs ( $p<0.0001)$, which were used to create the prognostic survival score. Specifically, patients belonging to scores $1,2,3,4$, and 5 had RHR values of $\leq 0.40,0.57-0.73,1.37,1.87$, and $>2.60$, respectively (Fig. 1A). The score performance in predicting death was defined by a Harrell's c-index of 0.75 (95\% CI 0.76-0.81). Subsequently, to investigate the impact of $I D H 1 / 2$ mutation on OS, the CART model was applied to $I D H 1 / 2$ wildtype EGBM patients (239 cases). A score from 1 to 4 was obtained from the 4 terminal nodes (Harrell's c-index of 0.74, 95\% CI 0.69-0.78; Fig. 1B). The 1-year estimated OS was computed for each score category (Tables 3 and 4). Overall, to facilitate the visualization of the survival analysis stratified by the score groups resulting from the CART models, Kaplan-Meier curves were generated (Fig. 2).

\section{Discussion}

In this retrospective investigation based on 322 elderly cases with newly diagnosed GBM, OS was analyzed based on the stratification of clinical, radiological, and molecular variables. The key findings for consideration were as follows: 1) age, volumetric tumor MRI pattern (expressed by the preoperative T1/T2 MRI index), EOR, and MGMT methylation status were confirmed as independent survival predictors on multivariate Cox regression analysis; 2) a novel prognostic score for EGBM surgical patients was assessed by CART analysis; and 3) surgery can be considered as a first therapeutic option in the workflow of EGBM patients, especially when the preoperative estimated EOR is greater than $80 \%$. 
TABLE 1. Clinical, radiological, molecular, surgical, and follow-up characteristics of the study population

\begin{tabular}{|c|c|}
\hline Parameter & Value \\
\hline No. of pts & 322 \\
\hline Mean age $\pm S D$, yrs & $72.28 \pm 4.86$ \\
\hline \multicolumn{2}{|l|}{ Sex, $n(\%)$} \\
\hline Female & $137(42.55)$ \\
\hline Male & $185(57.45)$ \\
\hline \multicolumn{2}{|l|}{ Side, $n(\%)$} \\
\hline $\mathrm{Lt}$ & $170(52.79)$ \\
\hline Rt & $152(47.21)$ \\
\hline \multicolumn{2}{|l|}{ Tumor site, $\mathrm{n}(\%)$} \\
\hline Precentral & $112(34.78)$ \\
\hline Postcentral & $100(31.06)$ \\
\hline Temporal + insular & $110(34.16)$ \\
\hline \multicolumn{2}{|l|}{ Clinical presentation, $\mathrm{n}(\%)$} \\
\hline No deficits & $14(4.35)$ \\
\hline Nonspecific symptoms (headache, nausea, vomiting, disorientation) & $108(33.54)$ \\
\hline Motor deficits & $76(23.6)$ \\
\hline Sensory deficits & $7(2.17)$ \\
\hline Visual/speech deficits & $64(19.88)$ \\
\hline Seizures & $53(16.46)$ \\
\hline Median preop KPS score (range) & $90(60-100)$ \\
\hline \multicolumn{2}{|l|}{ Preop, n (\%) } \\
\hline Diabetes & $32(9.94)$ \\
\hline Solid tumor & $25(7.77)$ \\
\hline Chronic pulmonary disease & $24(7.45)$ \\
\hline Peptic ulcer disease & $15(4.66)$ \\
\hline Previous myocardial infarction & $12(3.73)$ \\
\hline Chronic kidney disease & $9(2.79)$ \\
\hline Cerebrovascular disease & $8(2.48)$ \\
\hline Mild liver disease & $6(1.86)$ \\
\hline Lymphoma/leukemia & $5(1.55)$ \\
\hline Connective tissue disease & $3(0.93)$ \\
\hline Metastatic tumor in recent history & $2(0.62)$ \\
\hline \multicolumn{2}{|l|}{ Preop CCl, n (\%)* } \\
\hline 0 & $150(50.17)$ \\
\hline 1 & $82(27.42)$ \\
\hline 2 & $52(17.39)$ \\
\hline$\geq 3$ & $15(5.01)$ \\
\hline \multicolumn{2}{|l|}{ Radiological features } \\
\hline Ependymal involvement, yes vs no & $114(35.4 \%)$ vs $208(64.6 \%)$ \\
\hline Corpus callosum involvement, yes vs no & $96(29.81 \%)$ vs $226(70.19 \%)$ \\
\hline Necrotic-cystic component, yes vs no & $248(77.02 \%)$ vs $74(22.98 \%)$ \\
\hline Midline shift, yes vs no & $151(46.89 \%)$ vs $171(53.11 \%)$ \\
\hline $\begin{array}{l}\text { Median preop tumor volume on postcontrast T1-weighted images } \\
\text { (range), } \mathrm{cm}^{3}\end{array}$ & $31.45(0.39-197.7)$ \\
\hline Median preop tumor volume on T2-weighted images (range), $\mathrm{cm}^{3}$ & $56.5(0.52-231)$ \\
\hline
\end{tabular}


» CONTINUED FROM PAGE 4

TABLE 1. Clinical, radiological, molecular, surgical, and follow-up characteristics of the study population

\begin{tabular}{|c|c|}
\hline Parameter & Value \\
\hline \multicolumn{2}{|l|}{ Preop T1/T2 MRI index, n (\%) } \\
\hline$<0.73$ & $185(57.45)$ \\
\hline$\geq 0.73$ & $137(42.55)$ \\
\hline Median residual tumor (range), $\mathrm{cm}^{3}$ & $1.344(0-191)$ \\
\hline Median EOR (range), continuous variable & $95(35-100)$ \\
\hline \multicolumn{2}{|l|}{ EOR, n (\%), categorical variable } \\
\hline $100 \%$ & $125(38.82)$ \\
\hline $90 \%-99 \%$ & $92(28.57)$ \\
\hline $80 \%-89 \%$ & $43(13.35)$ \\
\hline$\leq 79 \%$ & $62(19.25)$ \\
\hline \multicolumn{2}{|l|}{ Biological features } \\
\hline MGMT met (yes vs no)† & $155(54.96 \%)$ vs 127 (45.04\%) \\
\hline IDH1/2 mutation (yes vs no) $\ddagger$ & $11(3.62 \%)$ vs $293(96.38 \%)$ \\
\hline Median Ki-67 \% (range) & $25(3-90)$ \\
\hline \multicolumn{2}{|l|}{ Two-gene model, $\mathrm{n}(\%) \S$} \\
\hline MGMT met \& IDH1/2 mutation & $6(2.16)$ \\
\hline MGMT met \& IDH1/2 wt & $149(53.60)$ \\
\hline MGMT unmet \& IDH1/2 mutation & $5(1.80)$ \\
\hline MGMT unmet \& IDH1/2 wt & $118(42.45)$ \\
\hline Median hospitalization (range), days & $8(5-14)$ \\
\hline \multicolumn{2}{|l|}{ Postop course, $n(\%)$} \\
\hline No deficits & $152(47.2)$ \\
\hline $\begin{array}{l}\text { Nonspecific postop symptoms (headache, nausea, vomiting, disori- } \\
\text { entation) }\end{array}$ & $44(13.66)$ \\
\hline Motor deficits & $73(22.67)$ \\
\hline Sensory deficits & $3(0.93)$ \\
\hline Visual/speech deficits & $46(14.29)$ \\
\hline Seizures & $4(1.24)$ \\
\hline \multicolumn{2}{|l|}{ Postop protocol, n (\%) } \\
\hline Stupp protocol & $250(77.64)$ \\
\hline CT or RT alone & $50(15.53)$ \\
\hline No adjuvant treatment & $22(6.83)$ \\
\hline \multicolumn{2}{|l|}{ 6-month follow-up, n (\%)ף } \\
\hline No deficits & $176(73.64)$ \\
\hline Motor deficits & $35(14.64)$ \\
\hline Sensory deficits & $8(3.35)$ \\
\hline Visual/speech deficits & $18(7.53)$ \\
\hline Seizures & $2(0.84)$ \\
\hline Median preop KPS score (range) & $90(60-100)$ \\
\hline OS (alive vs dead) & 77 (23.91\%) vs $245(76.09 \%)$ \\
\hline OS at 1-yr follow-up & $42.07 \%$ \\
\hline OS at 2-yr follow-up & $14.89 \%$ \\
\hline PFS (no recurrence vs recurrence) & $52(16.15 \%)$ vs $270(83.85 \%)$ \\
\hline PFS at 1-year follow-up & $24.8 \%$ \\
\hline PFS at 2-year follow-up & $9.64 \%$ \\
\hline
\end{tabular}




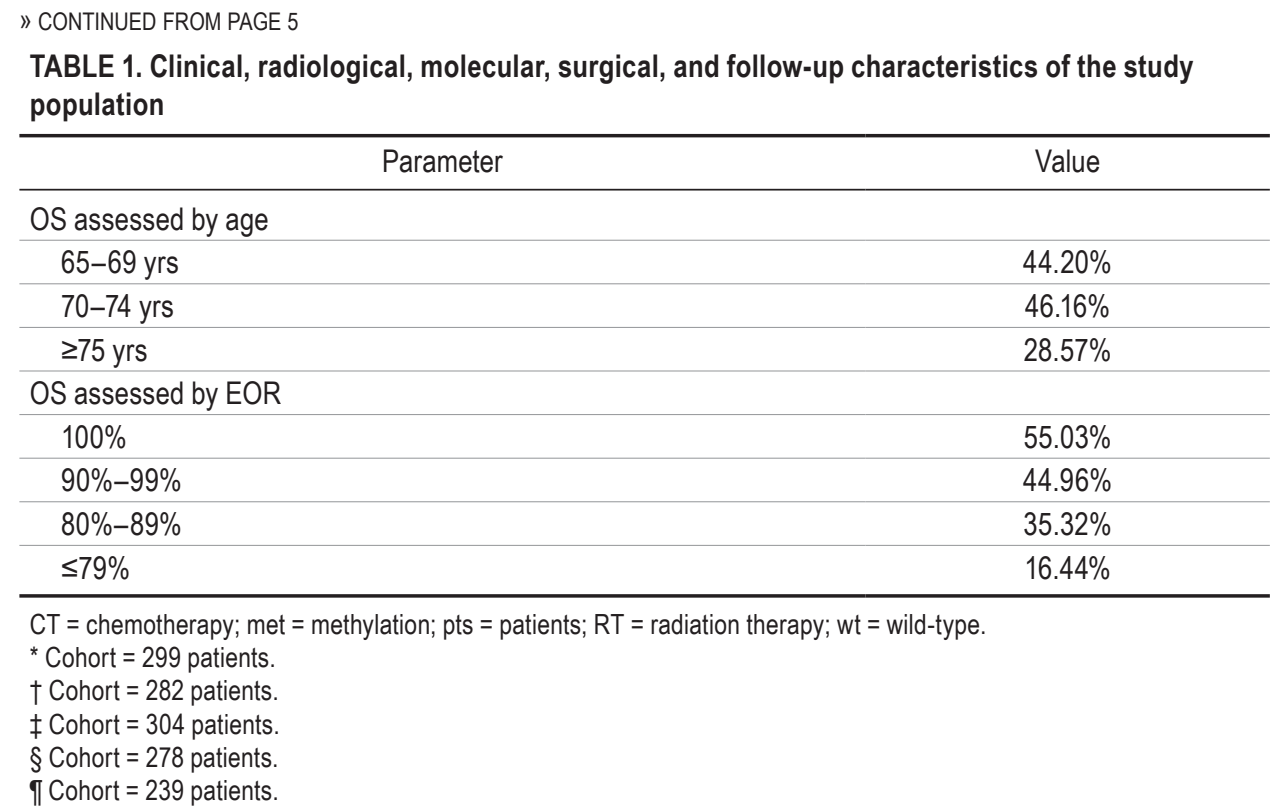

\section{GBM in the Elderly}

In the last decade, the role of surgery has been shown to be the primary option in GBM management, especially in light of recent literature demonstrating a survival benefit associated with a greater EOR. ${ }^{8,21,25,28-36}$ The incidence of intracranial tumors in elderly individuals is increasing due to aging of the population and increasing life expectancy. ${ }^{37}$

In 2017, according to Eurostat, the European Union population over the age of 65 years has an additional 20 years of life expectancy, and the percentage of people older than 80 years is expected to more than double in the coming decades, thus unavoidably changing the shape of the age pyramid. These age-associated demographic trends are already having a significant impact with regard to modifications in the disease epidemiology and management of neurosurgical care. Unfortunately, EGBM patients tend to have a drastically reduced survival compared with their younger counterparts. ${ }^{1,38,39}$ This could partially be explained by unfavorable tumor biology, performance status, comorbidities, treatment toxicity, trend toward less aggressive treatment, etc. Taking into account the poor prognosis and progressive increase in the incidence of GBM in the elderly population, investigation of treatment efficiency is of significant interest in the management of these patients ${ }^{10,11,13-19}$ (Table 5, Fig. 3).

\section{Survival Analysis in EGBM}

This retrospective investigation supports the widely known role of age, tumor preoperative MRI index, EOR, and MGMT methylation status as independent predictors of survival. Our results confirmed the poorer prognosis for EGBM patients with increasing age. ${ }^{1,6,40}$ When stratifying the survival results according to age intervals, we found that 1-year OSs in subgroups of patients who were 65-69, $70-74,75-79$, and $\geq 80$ years old were $42.20 \%, 46.16 \%$, $28.57 \%$, and $4.26 \%$, respectively.
Although several investigations have found that extensive resection is associated with longer survival in EGBM patients, aggressive surgery remains a controversial issue, mainly due to concerns over the balance between treatment benefits and side effects based on age, comorbidity conditions, and supposed different tumor biologies. . $^{10,11,13,14,16,17,19}$ With regard to the role of EOR, several retrospective investigations showed that greater EOR appears to correlate with an incremental OS benefit in the elderly population, similar to younger patients. . $^{10,11,13,14,16,17,19}$

Despite previous investigations recognizing EOR as an independent survival predictor, volumetric data were analyzed as qualitative and not quantitative variables. In this study, we reported the quantitative data and identified a threshold value capable of discriminating the survival benefit. The volumetric analysis showed 1-year survival rates of $55.03 \%, 44.96 \%, 35.32 \%$, and $16.44 \%$, when the EOR was $100 \%, 90 \%-99 \%, 80 \%-89 \%$, and $\leq 79 \%$, respectively. In addition, infiltration of the corpus callosum caused a worse prognosis $(\mathrm{p}=0.023)$ as an indirect measure of the possibility of obtaining radical resection in consideration of the vast tumor infiltration.

Our study also noted the role of $M G M T$ even among EGBM patients $(\mathrm{p}=0.002)$, which was consistent with other investigations. ${ }^{16,17}$ Concerning the radiological data, our results confirmed the prognostic survival value of the preoperative T1/T2 MRI index, clarifying its role in predicting a more aggressive biological behavior in those cases with a value close to $1(\mathrm{p}=0.002)$. In view of the wide heterogeneity of GBM, combining next-generation sequence analysis and assessments of MRI texture analysis parameters could further clarify the role of this volumetric index. ${ }^{21,41}$

Interestingly, unlike other studies, no correlation between KPS score $(p=0.254)$ and CCI score $(p=0.574)$ and OS was found. ${ }^{1,37}$ This could be explained by the fact that in the present investigation, patients treated surgically had 
TABLE 2. Predictors of OS in univariate and multivariate analyses

\begin{tabular}{|c|c|c|c|c|c|c|}
\hline \multirow[b]{2}{*}{ Variable } & \multicolumn{3}{|c|}{ Univariate Analysis } & \multicolumn{3}{|c|}{ Multivariate Analysis } \\
\hline & $\mathrm{HR}$ & $95 \% \mathrm{Cl}$ & $p$ Value & $\mathrm{HR}$ & $95 \% \mathrm{Cl}$ & p Value \\
\hline Age, yrs & 1.028 & $1.001-1.055$ & 0.035 & 1.040 & $1.006-1.075$ & 0.018 \\
\hline \multicolumn{7}{|l|}{ Sex } \\
\hline Male & 1 & & & & & \\
\hline Female & 1.161 & $0.901-1.497$ & 0.247 & & & \\
\hline \multicolumn{7}{|l|}{ Side } \\
\hline $\mathrm{Lt}$ & 1 & & & & & \\
\hline Rt & 1.169 & $0.908-1.506$ & 0.224 & & & \\
\hline \multicolumn{7}{|l|}{ Tumor site } \\
\hline Precentral & 1 & & & & & \\
\hline Retrocentral & 0.987 & $0.723-1.348$ & 0.938 & & & \\
\hline Temporal + insular & 1.026 & $0.758-1.389$ & 0.866 & & & \\
\hline Preop CCI (0 vs $\geq 1)$ & 0.862 & $0.664-1.119$ & 0.265 & & & \\
\hline Preop KPS score & 1.112 & $0.905-1.110$ & 0.574 & & & \\
\hline \multicolumn{7}{|l|}{ Radiological features } \\
\hline Ependymal involvement (yes vs no) & 1.132 & $0.900-1.425$ & 0.286 & & & \\
\hline Corpus callosum involvement (yes vs no) & 1.308 & $0.998-1.713$ & 0.040 & 1.449 & $1.051-1.998$ & 0.023 \\
\hline Necrotic-cystic component (yes vs no) & 0.980 & $0.727-1.322$ & 0.897 & & & \\
\hline Midline shift (yes vs no) & 1.057 & $0.822-1.360$ & 0.664 & & & \\
\hline $\begin{array}{l}\text { Preop tumor volume on postcontrast T1-weighted } \\
\text { images, } \mathrm{cm}^{3}\end{array}$ & 1.000 & $0.998-1.002$ & 0.500 & & & \\
\hline Preop tumor volume on T2-weighted images, $\mathrm{cm}^{3}$ & 0.994 & $0.992-0.997$ & $<0.0001$ & 0.997 & $0.993-1.000$ & 0.123 \\
\hline Preop T1/T2 MRI index & 5.408 & $3.144-9.301$ & $<0.0001$ & 3.206 & $1.537-6.685$ & 0.002 \\
\hline Residual tumor, $\mathrm{cm}^{3}$ & 1.016 & $1.009-1.022$ & $<0.0001$ & 1.018 & $0.997-1.040$ & 0.081 \\
\hline EOR (continuous variable) & 0.983 & $0.978-0.987$ & $<0.0001$ & 0.985 & $0.977-0.994$ & 0.002 \\
\hline \multicolumn{7}{|l|}{ EOR (categorical variable) } \\
\hline $100 \%$ & 1 & & & & & \\
\hline $90 \%-99 \%$ & 1.296 & 0.944-1.799 & 0.108 & & & \\
\hline $80 \%-89 \%$ & 1.774 & $1.190-2.644$ & 0.005 & & & \\
\hline$\leq 79 \%$ & 3.424 & $2.412-4.861$ & $<0.0001$ & & & \\
\hline \multicolumn{7}{|l|}{ Biological features } \\
\hline MGMT methylation (yes vs no) & 0.645 & $0.490-0.849$ & 0.002 & 0.678 & $0.500-0.919$ & 0.012 \\
\hline IDH1/2 mutation (yes vs no) & 0.476 & $0.223-1.014$ & 0.055 & 0.658 & $0.287-1.512$ & 0.325 \\
\hline Ki-67 & 1.002 & $0.995-1.010$ & 0.456 & & & \\
\hline \multicolumn{7}{|l|}{ Two-gene model } \\
\hline MGMT met \& IDH1/2 mut & 1 & & & & & \\
\hline MGMT met \& IDH1/2 wt & 1.740 & $0.639-4.736$ & 0.278 & & & \\
\hline MGMT unmet \& IDH1/2 mut & 1.183 & $0.264-5.298$ & 0.826 & & & \\
\hline MGMT unmet \& IDH1/2 wt & 2.788 & $1.020-7.618$ & 0.045 & & & \\
\hline
\end{tabular}

Boldface type represents statistically significant results $(p<0.05)$.

high KPS scores with chronic yet stabilized comorbidities, while those with relevant morbidities were excluded in the preoperative anesthesiological evaluation. Patients with a preoperative KPS score $<60$ or chronic uncontrolled diseases underwent only needle biopsy and, consequently, were excluded from this investigation.

Flanigan et al. ${ }^{1}$ reported that patients with a CCI score $\geq 1$ were associated with decreased survival $(p=0.018)$. The difference obtained in identifying CCI score as a survival prognostic factor in EGBM patients could be due to the presence or absence of chronic comorbidities in addition to the specific therapeutic controls and treatments. This suggests the need for more adequate scales to assess the severity of comorbidities, in addition to other chronic pathologies, in patients with GBM.

Overall, these results emphasize the importance of a multidisciplinary approach for a careful evaluation of surgical options in EGBM patients. 
lus et al.

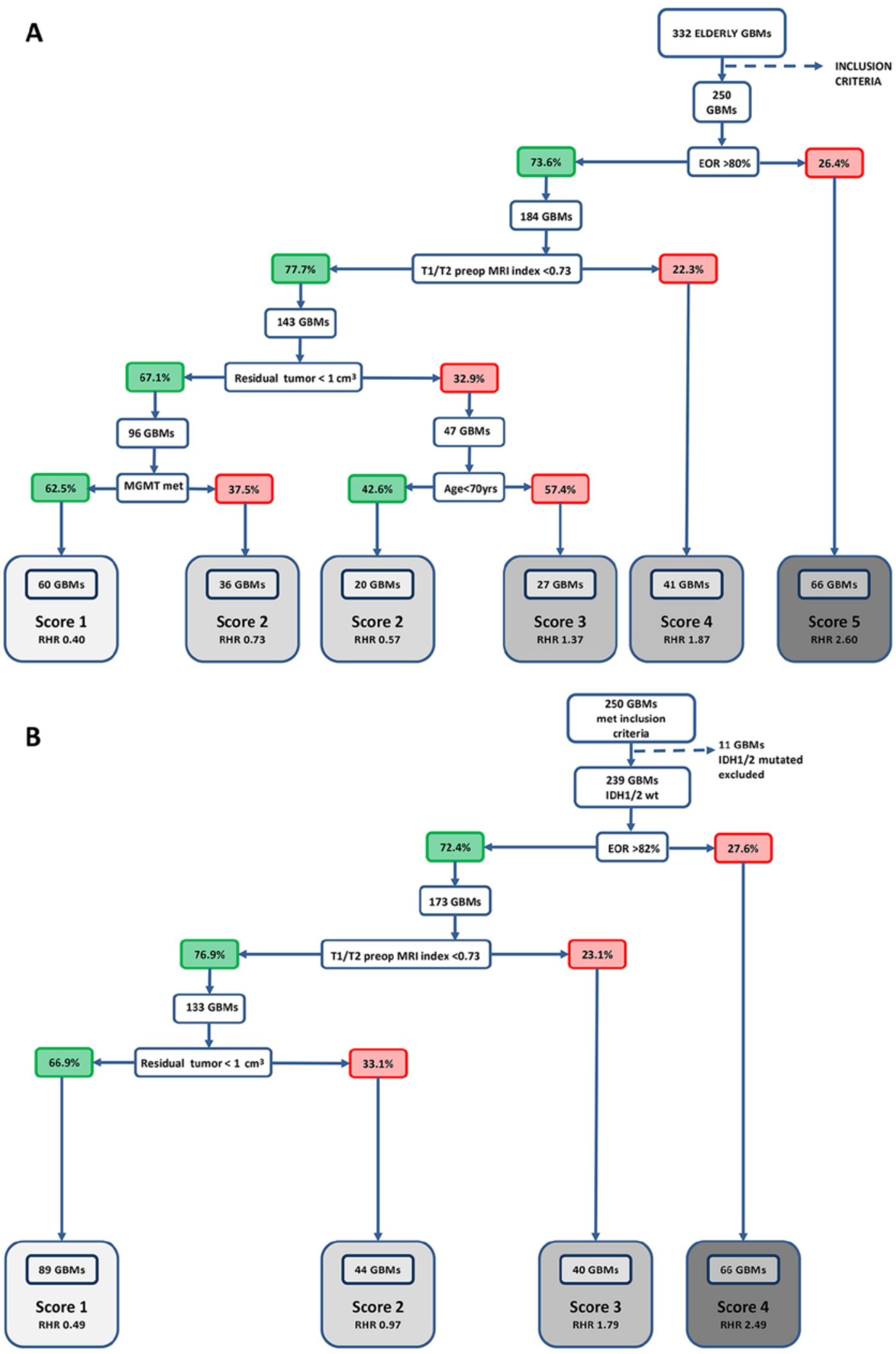

FIG. 1. Random forest, CART algorithm. The CART algorithm provides a graphic visualization of the interaction between risk factors detected by Cox survival analysis. In each hierarchical node, the study population is split according to the presence (green) or not (red) of the variable able to influence prognosis. A: CART model performed on 250 EGBM cases that met all the selection criteria. A score from 1 to 5 was assigned to the 6 terminal nodes thus defined based on the RHR. Patients belonging to the subgroup with $R H R=0.73$ and to the subgroup with $R H R=0.55$ were joined to create a single group, labeled "score 2." This was done considering the small sample size of each subgroup and similarities in RHR values. B: CART model applied to IDH1/2 wild-type EGBM patients (239 cases). A score from 1 to 4 was obtained from the 4 terminal nodes. 
TABLE 3. Estimated OS at 12, 18, and 24 months in EGBM patients $(n=250)$ according to the CART score

\begin{tabular}{|c|c|c|c|c|c|c|c|}
\hline \multirow[b]{2}{*}{ Score* $^{*}$} & \multirow[b]{2}{*}{ Variable } & \multirow[b]{2}{*}{$\mathrm{HR}$} & \multirow[b]{2}{*}{$95 \% \mathrm{Cl}$} & \multirow[b]{2}{*}{ p Value } & \multicolumn{3}{|c|}{ Estimated OS (\%) } \\
\hline & & & & & $12 \mathrm{mos}$ & $18 \mathrm{mos}$ & 24 mos \\
\hline 1 & $\begin{array}{l}\text { EOR }>80 \% \text {, preop T1/T2 MRI index }<0.73 \text {, resid- } \\
\text { ual tumor }<1 \mathrm{~cm}^{3}, M G M T \text { met }\end{array}$ & 1 & - & - & 76.73 & 56.30 & 44.45 \\
\hline \multirow[t]{2}{*}{2} & $\begin{array}{l}\text { EOR }>80 \% \text {, preop T1/T2 MRI index }<0.73 \text {, resid- } \\
\quad \text { ual tumor }<1 \mathrm{~cm}^{3}, \text { MGMT unmet }\end{array}$ & 1.626 & $0.985-2.685$ & 0.058 & 69.00 & 48.53 & 15.25 \\
\hline & $\begin{array}{l}\text { EOR }>80 \% \text {, preop T1/T2 MRI index }<0.73 \text {, resid- } \\
\text { ual tumor }>1 \mathrm{~cm}^{3} \text {, age }<70 \text { yrs }\end{array}$ & & & & & & \\
\hline 3 & $\begin{array}{l}\text { EOR }>80 \% \text {, preop T1/T2 MRI index }<0.73 \text {, resid- } \\
\quad \text { ual tumor }>1 \mathrm{~cm}^{3} \text {, age }>70 \mathrm{yrs}\end{array}$ & 3.290 & $1.875-5.775$ & 0.000 & 40.74 & 12.07 & 6.04 \\
\hline 4 & EOR $>80 \%$, preop T1/T2 MRI index $>0.73$ & 4.743 & $2.829-7.954$ & 0.000 & 15.80 & 3.95 & - \\
\hline 5 & EOR $<80 \%$ & 7.529 & $4.712-12.031$ & 0.000 & 15.21 & 3.04 & - \\
\hline
\end{tabular}

Boldface type represents statistically significant results $(p<0.05)$.

${ }^{*}$ A survival score from 1 to 5 was defined based on CART analysis.

TABLE 4. Estimated OS at 12, 18, and 24 months in EGBM patients with IDH1/2 wild-type $(n=239)$ according to the CART score

\begin{tabular}{|c|c|c|c|c|c|c|c|}
\hline \multirow[b]{2}{*}{ Score $^{*}$} & \multirow[b]{2}{*}{ Variable } & \multirow[b]{2}{*}{$\mathrm{HR}$} & \multirow[b]{2}{*}{$95 \% \mathrm{Cl}$} & \multirow[b]{2}{*}{ p Value } & \multicolumn{3}{|c|}{ Estimated OS (\%) } \\
\hline & & & & & $12 \mathrm{mos}$ & $18 \mathrm{mos}$ & $24 \mathrm{mos}$ \\
\hline 1 & $\begin{array}{l}\text { EOR }>82 \% \text {, preop T1/T2 MRI index }<0.73 \text {, residual tumor } \\
\quad<1 \mathrm{~cm}^{3}\end{array}$ & 1 & - & - & 72.90 & 53.38 & 32.52 \\
\hline 2 & $\begin{array}{l}\text { EOR }>82 \% \text {, preop T1/T2 MRI index }<0.73 \text {, residual tumor } \\
\quad>1 \mathrm{~cm}^{3}\end{array}$ & 1.914 & $1.216-3.013$ & 0.005 & 49.72 & 22.90 & 11.45 \\
\hline 3 & EOR $>82 \%$, preop T1/T2 MRI index $>0.73$ & 3.622 & $2.300-5.703$ & $<0.0001$ & 16.25 & 4.06 & - \\
\hline 4 & EOR $<82 \%$ & 5.772 & $3.875-8.596$ & $<0.0001$ & 15.21 & - & - \\
\hline
\end{tabular}

Boldface type represents statistically significant results $(p<0.05)$.

${ }^{*}$ A survival score from 1 to 4 was defined based on CART analysis.

A

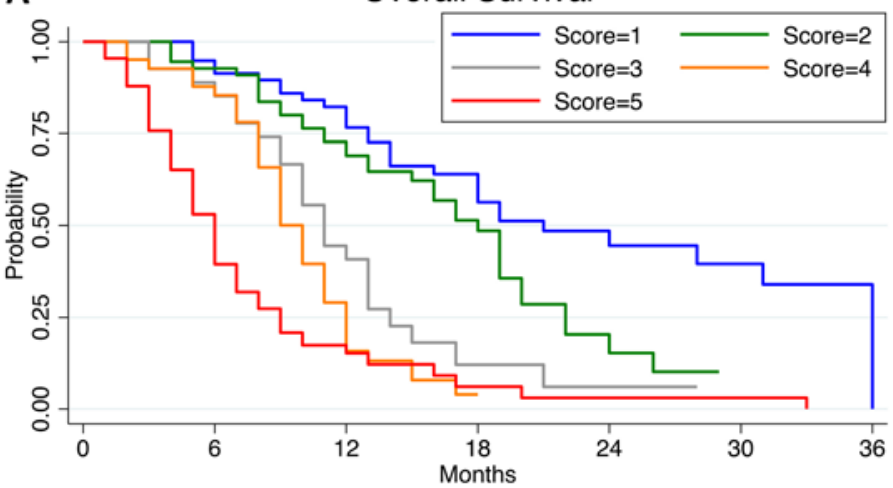

B

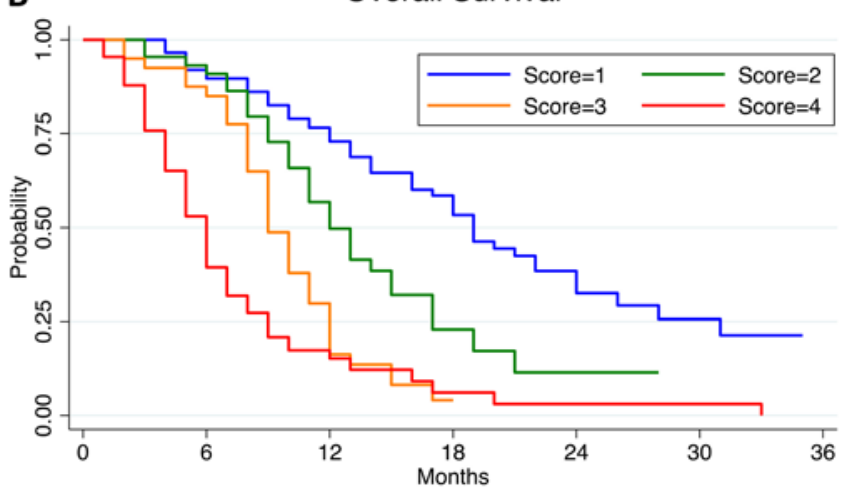

FIG. 2. OS stratified by CART analysis prognostic score. Kaplan-Meier curves display the OS of EGBM patients according to the prognostic scores elaborated by the CART model. A: Survival stratified by the score from 250 EGBM cases that met the selection criteria. B: Survival stratified by the score from 239 EGBM IDH1/2 wild-type cases. 


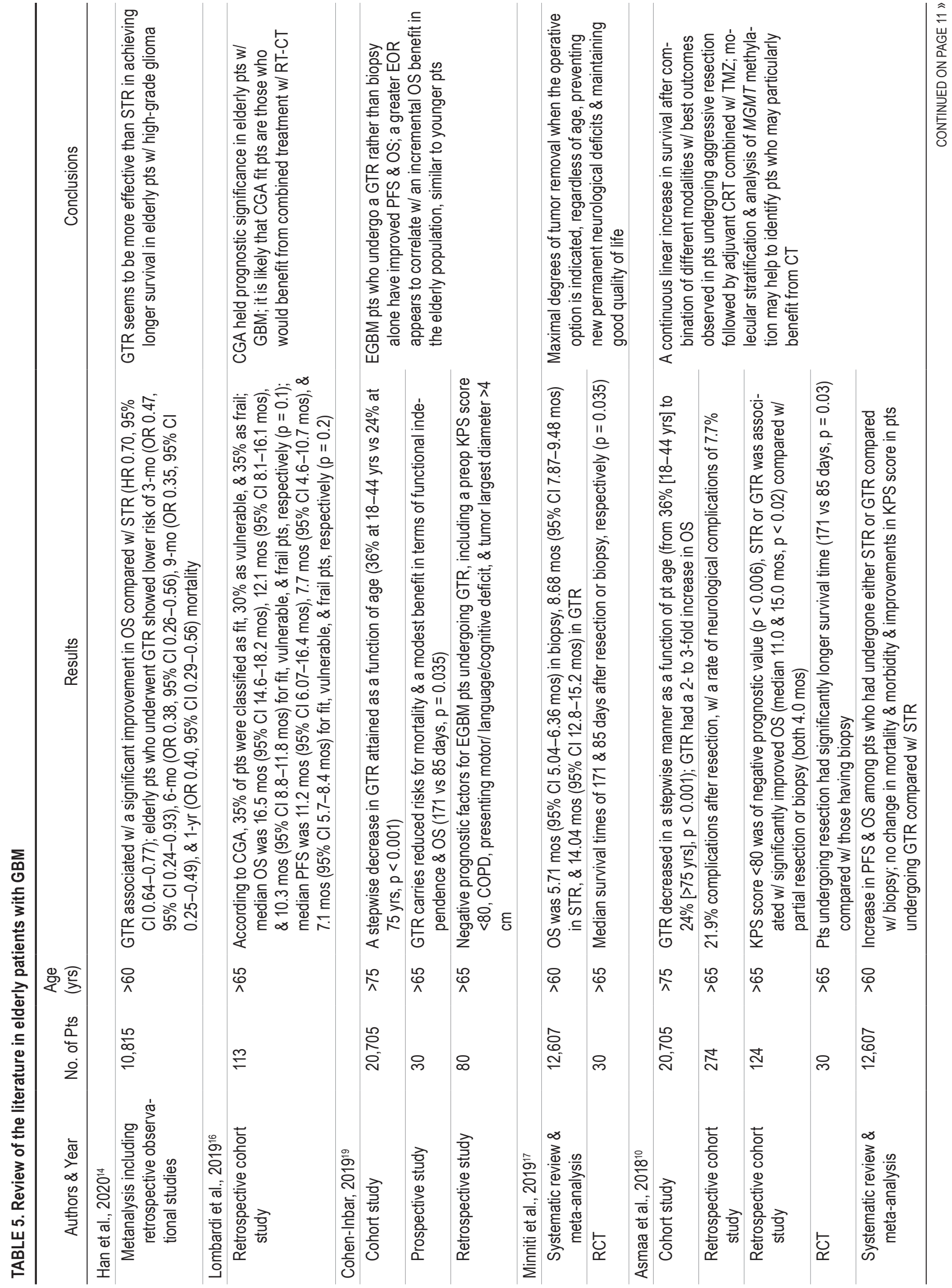




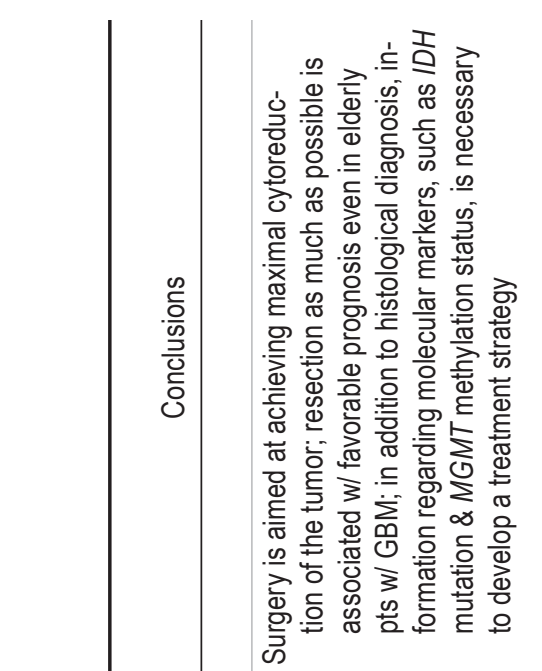

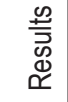

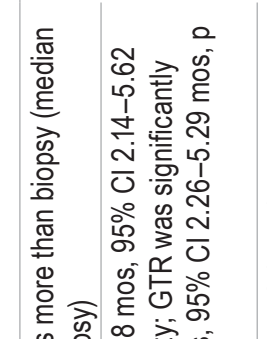

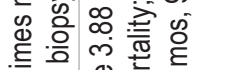

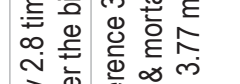

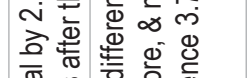

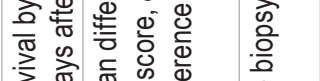

帘贾造

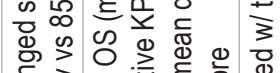

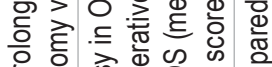

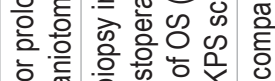

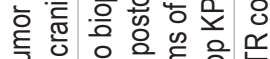

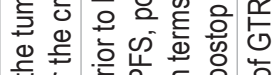

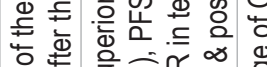

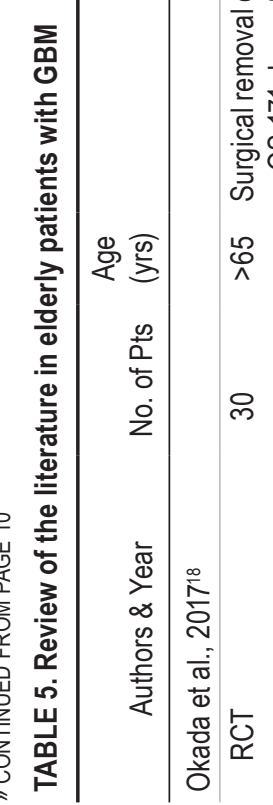

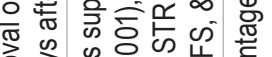

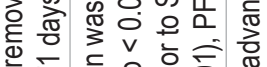

는든 든 응 흥 $\frac{\bar{\sigma}}{\pi}$

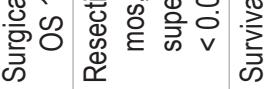

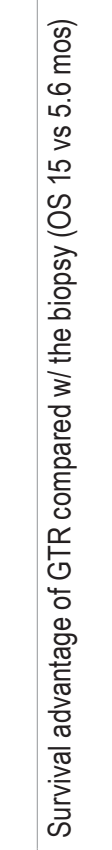

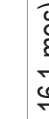

बू

잉

$2 \sqrt[2]{2}$

它

O

\begin{tabular}{l|l}
$\frac{x}{5}$ & $\frac{x}{5}$ \\
$\frac{5}{3}$ & $\frac{5}{3}$ \\
\end{tabular}

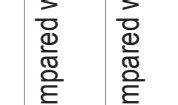

हे हे

品哭

¿

宽

$\frac{\pi}{\pi} \quad \sum^{\frac{\pi}{\pi}}$

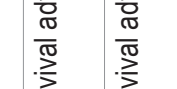

œ

$\begin{array}{llll}0 & 0 & 0\end{array}$

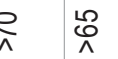

㟔 苔

ڤิ̀

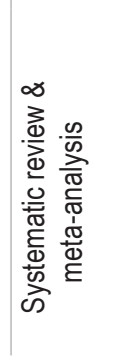

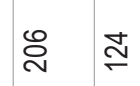

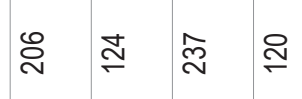

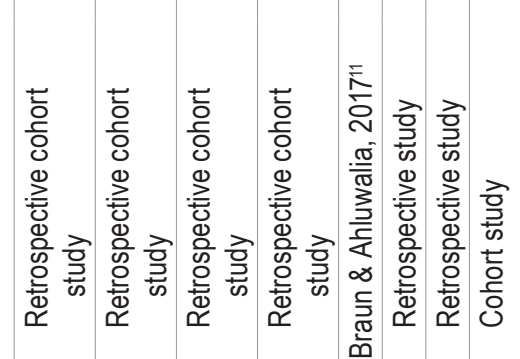

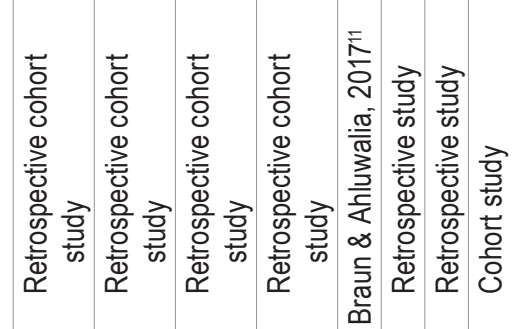

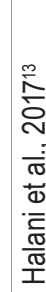

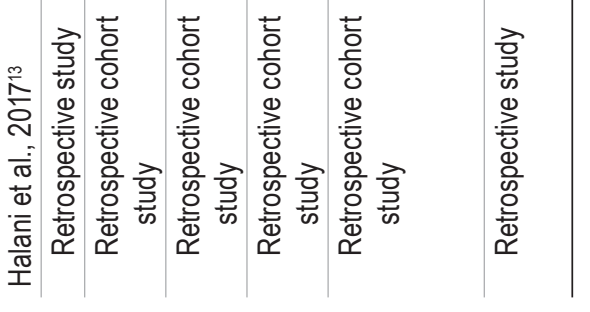

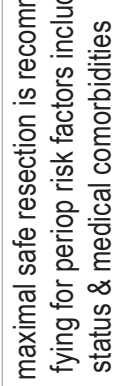

这

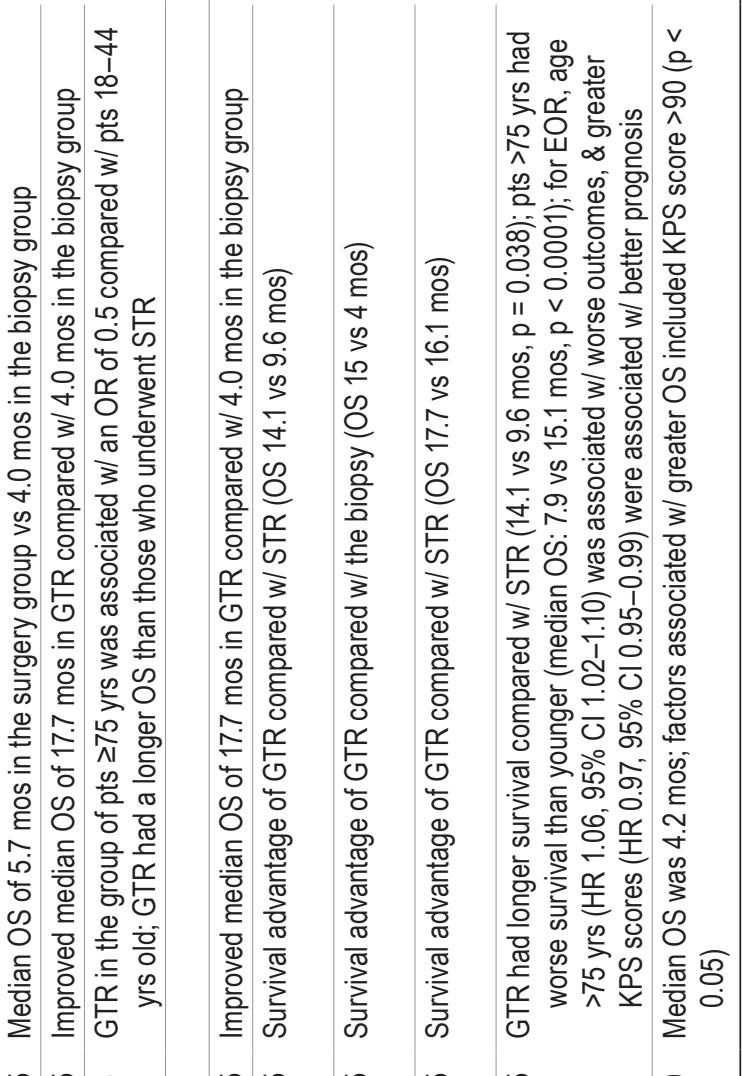

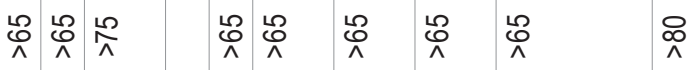

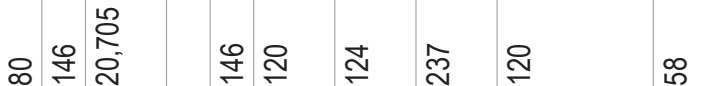




\section{CART Analysis}

The principal novelty of this study is the elaboration of a prognostic and integrated score based on the CART analysis. This approach identifies the strongest prognostic variables and generates a creative and integrative visualization chart that illustrates the results in an easily interpretable tree structure from a large number of data. The model is based on the influence of interactions of various parameters and not on single ones, which permits the creation of a model that closely reflects the disease complexity. In this investigation, the CART analysis based on the 250 cases provided 6 terminal nodes, the RHRs of which were used to generate the prognostic score with the purpose of facilitating the survival stratification before patients were discharged postoperatively.

Patients belonging to the subgroups with RHR $=0.73$ and RHR $=0.55$ were joined to create a single group labeled "score 2." This was done because of the small sample size of each subgroup and similarities in RHR values. Patients with scores of 1 or 2 had better survival, with 1-year estimated OSs of $76.73 \%$ and $69.00 \%$, respectively. The worst survival was for patients with scores from 3 to 5, with 1-year estimated OS after surgery ranging between $40.74 \%$ and $15.21 \%$ after surgery.

It is important to highlight that the EOR was the most robust influencing factor in the algorithm hierarchy, while age appeared at the third node of the CART algorithm, thus strengthening the role of surgery also in EGBM and performing patients (who have a high preoperative KPS score), when surgical planning allowed us to preoperatively estimate an EOR $>80 \% .^{6,17,42}$ In a previous investigation, Flanigan et al. elaborated a risk prognostic score in EGBM patients based on variables identified using the multivariate stepwise analysis (age, EOR, preoperative weakness, tumor size, and CCI). ${ }^{1}$ A point designation was then given to each factor and points were totaled for each patient, considering only the presence of the variables and ruling out their interactions. Our model thus provides for a rapid and accurate assessment of survival prognosis after surgery and relies on concrete parameters rather than on a subjective metric.

It is widely reported in the literature that the $I D H I / 2$ mutation is associated with a better prognosis in GBM patients in terms of disease-free survival and progression. IDHI/2 mutant GBMs represent less than $10 \%$ of all GBMs and show different genetic, epigenetic, and clinical features compared with the $1 / 2$ wild-type counterpart. ${ }^{43}$ In this investigation, the $I D H I / 2$ mutation was detected in only $3.4 \%$ of cases (11 patients), with a similar distribution within each score class identified by CART analysis (3, 1, 2, 3, and $2 I D H I / 2$ EGBM patients for scores 1, 2, 3, 4, and 5, respectively). The equal portioning of the IDHI/2 mutation may determine that its positive survival impact is equally distributed within the different score groups identified by CART analysis.

Analyzing only IDHI/2 wild-type EGBM patients, we excluded age and $M G M T$ methylation status from the model, while the EOR, residual tumor, and preoperative neuroradiological tumor growth pattern, expressed by the preoperative T1/T2 MRI index, were confirmed as the fundamental nodes in the prognostic model. Generally, 


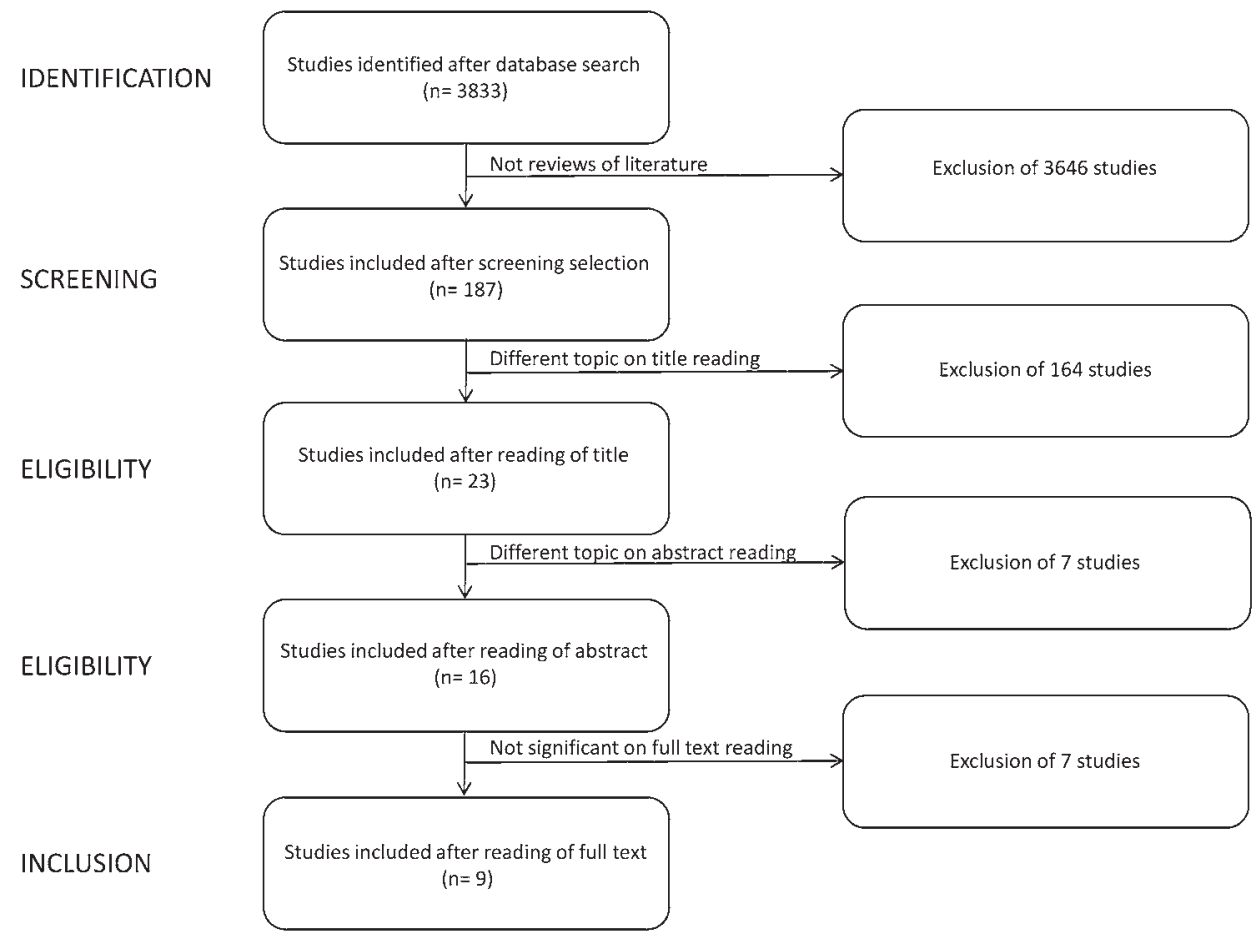

FIG. 3. Systematic review and meta-analysis flowchart. The figure shows the different decisional phases regarding the inclusion and exclusion of the papers reviewed in the studies based on the role of surgery in EGBM patients.

$I D H$-mutant gliomas are younger, and in these patients GBM is more likely to derive from a low-grade glioma and therefore also carries the IDH mutation. ${ }^{44}$ This investigation highlights that EOR is the main prognostic factor in EGBM patients, giving rise to the first split node in both generated CART models (Fig. 1). This may suggest that the OS is influenced by other numerous mutations, such as mutations in ATRX, CIC, EGFR, FUBPI, NOTCHI, PTEN, H3F3A, IDHI/2, PIK3CA, and BRAF, and amplifications in $E G F R$ or MDM2; copy number alterations of chromosomes 1p, 7, 10, and 19q are involved in glioma genesis and tumor progression..$^{41,45}$

\section{Limitations and Future Directions}

A limitation of our study is that we included only patients with resectable GBM based on clinical and radiological criteria (high preoperative KPS score, controlled comorbidities, and/or high chances of achieving a large EOR). Patients who underwent needle biopsy, who did not undergo resection, were thus excluded. An additional limitation is that comorbidities were not adequately discussed (heart disease, cancer, anticoagulation, etc.), because only patients with controlled mild or moderate comorbidities were considered for surgery. It is thus important to have stringent clinical selection criteria in EGBM patients to select which may benefit from surgery so that underlying comorbidities do not have a direct impact on surgical outcomes.

Despite the inherent limitations of the retrospective nature of this study, the prognostic score elaborated for EGBMs could be useful in a day-to-day clinical environ- ment. It could also prove to be useful after surgery and previous oncological treatments, to discuss prognosis and draw future prospective clinical trials. Patients with better OS showed a better PFS and lower score. ${ }^{21,45}$ The prognostic survival score assessed in this investigation can thus be considered an indirect measure of tumor progression.

An additional limitation is represented by the heterogeneous treatment at tumor recurrence. Each patient underwent individualized management at tumor progression. It is well known that to improve the prediction models, salvage treatment information should be updated in the analysis at the time of tumor progression. Moreover, the study lacks details about functional recovery time and neurocognitive outcomes according to variable levels of resection. To overcome this drawback, future prospective multicenter studies based on larger cohorts with longer follow-up periods need to include time-dependent analysis.

Future studies are needed to further assess the numerous molecular and genomic markers that may prove to be of clinical interest in GBM. In addition, radiological features should be included in prospective future clinical studies considering the growing importance of radiogenomics. Considering the heterogeneity of GBM, texture features from multiparametric MRI and next-generation sequence analysis could prove to be of assistance in managing these patients. Computed prognostic scores may prove to be useful in a day-to-day clinical setting and in research to provide more thorough assessments in future prospective clinical trials. In conclusion, the survival score could be useful when deciding and discussing prognosis to better address the entire management of EGBM patients. 


\section{Conclusions}

Elderly patients with GBM typically carry a poor prognosis. There are no gold standards or widespread guidelines to be applied to this group of patients, and optimal strategy and management remain debatable. Advanced age alone should not necessarily preclude optimal resection followed by adjuvant radiation and chemotherapy. Our study showed that prediction models can be used to generate a promising, thorough, and new clinical prognostic score for EGBM surgical patients to guide clinicians in the decision-making process. Thorough evaluation and selection of EGBM patients may lead to favorable survival benefit.

\section{Acknowledgments}

We would like to thank Mark Zeppieri, MD, PhD, for the scientific and English revision of the manuscript.

\section{References}

1. Flanigan PM, Jahangiri A, Kuang R, et al. Developing an algorithm for optimizing care of elderly patients with glioblastoma. Neurosurgery. 2018;82(1):64-75.

2. Chaichana KL, Chaichana KK, Olivi A, et al. Surgical outcomes for older patients with glioblastoma multiforme: preoperative factors associated with decreased survival. Clinical article. J Neurosurg. 2011;114(3):587-594.

3. Iwamoto FM, Cooper AR, Reiner AS, et al. Glioblastoma in the elderly: the Memorial Sloan-Kettering Cancer Center Experience (1997-2007). Cancer. 2009;115(16):3758-3766.

4. Babu R, Komisarow JM, Agarwal VJ, et al. Glioblastoma in the elderly: the effect of aggressive and modern therapies on survival. J Neurosurg. 2016;124(4):998-1007.

5. Scott JG, Suh JH, Elson P, et al. Aggressive treatment is appropriate for glioblastoma multiforme patients 70 years old or older: a retrospective review of 206 cases. Neuro Oncol. 2011;13(4):428-436.

6. Tanaka S, Meyer FB, Buckner JC, et al. Presentation, management, and outcome of newly diagnosed glioblastoma in elderly patients. J Neurosurg. 2013;118(4):786-798.

7. Álvarez de Eulate-Beramendi S, Álvarez-Vega MA, Balbin $\mathrm{M}$, et al. Prognostic factors and survival study in high-grade glioma in the elderly. Br J Neurosurg. 2016;30(3):330-336.

8. Molinaro AM, Hervey-Jumper S, Morshed RA, et al. Association of maximal extent of resection of contrast-enhanced and non-contrast-enhanced tumor with survival within molecular subgroups of patients with newly diagnosed glioblastoma. JAMA Oncol. 2020;6(4):495-503.

9. Mariniello G, Peca C, De Caro MDB, et al. Glioblastoma in the elderly: the impact of advanced age on treatment and survival. J Neurol Surg A Cent Eur Neurosurg. 2014;75(4): 276-281.

10. Asmaa A, Dixit S, Rowland-Hill C, et al. Management of elderly patients with glioblastoma-multiforme-a systematic review. Br J Radiol. 2018;91(1088):20170271.

11. Braun K, Ahluwalia MS. Treatment of glioblastoma in older adults. Curr Oncol Rep. 2017;19(12):81.

12. Cohen-Inbar O. Geriatric brain tumor management part I: meningioma. J Clin Neurosci. 2019;67:5-9.

13. Halani SH, Babu R, Adamson DC. Management of glioblastoma multiforme in elderly patients: a review of the literature. World Neurosurg. 2017;105:53-62.

14. Han Q, Liang H, Cheng P, et al. gross total vs. subtotal resection on survival outcomes in elderly patients with high-grade glioma: a systematic review and meta-analysis. Front Oncol. 2020;10:151.
15. Jordan JT, Gerstner ER, Batchelor TT, et al. Glioblastoma care in the elderly. Cancer. 2016;122(2):189-197.

16. Lombardi G, Bergo E, Caccese M, et al. Validation of the comprehensive geriatric assessment as a predictor of mortality in elderly glioblastoma patients. Cancers (Basel). 2019; 11(10):E1509.

17. Minniti G, Lombardi G, Paolini S. Glioblastoma in elderly patients: current management and future perspectives. Cancers (Basel). 2019;11(3):E336.

18. Okada M, Miyake K, Tamiya T. Glioblastoma treatment in the elderly. Neurol Med Chir (Tokyo). 2017;57(12):667-676.

19. Cohen-Inbar O. Geriatric brain tumor management part II: Glioblastoma multiforme. J Clin Neurosci. 2019;67:1-4.

20. Ganggayah MD, Taib NA, Har YC, et al. Predicting factors for survival of breast cancer patients using machine learning techniques. BMC Med Inform Decis Mak. 2019;19(1):48.

21. Ius T, Pignotti F, Della Pepa GM, et al. A novel comprehensive clinical stratification model to refine prognosis of glioblastoma patients undergoing surgical resection. Cancers (Basel). 2020;12(2):E386.

22. Chen W, Xie X, Wang J, et al. A comparative study of logistic model tree, random forest, and classification and regression tree models for spatial prediction of landslide susceptibility. Catena. 2017;151:147-160.

23. Senders JT, Staples PC, Karhade AV, et al. Machine learning and neurosurgical outcome prediction: a systematic review. World Neurosurg. 2018;109:476-486.e1.

24. Charlson M, Szatrowski TP, Peterson J, Gold J. Validation of a combined comorbidity index. J Clin Epidemiol. 1994; 47(11):1245-1251.

25. Ius T, Pignotti F, Della Pepa GM, et al. Glioblastoma: from volumetric analysis to molecular predictors. J Neurosurg Sci. Published online February 4, 2020. doi:10.23736/S03905616.20.04850-X

26. Yohannes Y, Hoddinott J. Classification and Regression Trees: An Introduction. International Food Policy Research Institute; 1999.

27. Schmid M, Wright MN, Ziegler A. On the use of Harrell's C for clinical risk prediction via random survival forests. $E x$ pert Syst Appl. 2016;63(C):450-459.

28. Li YM, Suki D, Hess K, Sawaya R. The influence of maximum safe resection of glioblastoma on survival in 1229 patients: Can we do better than gross-total resection? J Neurosurg. 2016;124(4):977-988.

29. Lacroix M, Abi-Said D, Fourney DR, et al. A multivariate analysis of 416 patients with glioblastoma multiforme: prognosis, extent of resection, and survival. J Neurosurg. 2001; 95(2):190-198.

30. Somma T, Ius T, Certo F, et al. From the champion to the team: new treatment paradigms in contemporary neurosurgery. World Neurosurg. 2019;131:141-148.

31. Ius T, Cesselli D, Isola M, et al. Combining clinical and molecular data to predict the benefits of carmustine wafers in newly diagnosed high-grade gliomas. Curr Treat Options Neurol. 2018;20(2):3.

32. Chaichana KL, Cabrera-Aldana EE, Jusue-Torres I, et al. When gross total resection of a glioblastoma is possible, how much resection should be achieved? World Neurosurg. 2014; 82(1-2):e257-e265.

33. Gessler F, Bernstock JD, Braczynski A, et al. Surgery for glioblastoma in light of molecular markers: impact of resection and MGMT promoter methylation in newly diagnosed IDH-1 wild-type glioblastomas. Neurosurgery. 2019;84(1): 190-197.

34. Illic R, Somma T, Savic D, et al. A survival analysis with identification of prognostic factors in a series of 110 patients with newly diagnosed glioblastoma before and after introduction of the Stupp regimen: a single-center observational study. World Neurosurg. 2017;104:581-588. 
35. Altieri R, Zenga F, Fontanella MM, et al. Glioma surgery: technological advances to achieve a maximal safe resection. Surg Technol Int. 2015;27:297-302.

36. Altieri R, Zenga F, Ducati A, et al. Tumor location and patient age predict biological signatures of high-grade gliomas. Neurosurg Rev. 2018;41(2):599-604.

37. Cloney M, D’Amico R, Lebovic J, et al. Frailty in geriatric glioblastoma patients: a predictor of operative morbidity and outcome. World Neurosurg. 2016;89:362-367.

38. Biau J, Chautard E, De Schlichting E, et al. Radiotherapy plus temozolomide in elderly patients with glioblastoma: a "real-life" report. Radiat Oncol. 2017;12(1):197.

39. Arvold ND, Cefalu M, Wang Y, et al. Comparative effectiveness of radiotherapy with vs. without temozolomide in older patients with glioblastoma. J Neurooncol. 2017;131(2): 301-311.

40. Sherrod BA, Gamboa NT, Wilkerson C, et al. Effect of patient age on glioblastoma perioperative treatment costs: a value driven outcome database analysis. J Neurooncol. 2019; 143(3):465-473.

41. Yang Y, Yan LF, Zhang X, et al. Optimizing texture retrieving model for multimodal MR image-based support vector machine for classifying glioma. J Magn Reson Imaging. 2019;49(5):1263-1274.

42. Cunha MLVD, Esmeraldo ACS, Henriques LAW, et al. Elderly patients with glioblastoma: the impact of the surgical resection extent on survival. Rev Assoc Med Bras (1992). 2019;65(7):937-945.

43. Li KK-W, Shi Z-F, Malta TM, et al. Identification of subsets of $I D H$-mutant glioblastomas with distinct epigenetic and copy number alterations and stratified clinical risks. $\mathrm{Neu}$ rooncol Adv. 2019;1(1):vdz015.

44. Louis DN, Perry A, Reifenberger G, et al. The 2016 World Health Organization Classification of Tumors of the Central Nervous System: a summary. Acta Neuropathol. 2016;131(6): 803-820.
45. Gorlia T, Stupp R, Brandes AA, et al. New prognostic factors and calculators for outcome prediction in patients with recurrent glioblastoma: a pooled analysis of EORTC Brain Tumour Group phase I and II clinical trials. Eur J Cancer. 2012;48(8):1176-1184.

\section{Disclosures}

The authors report no conflict of interest concerning the materials or methods used in this study or the findings specified in this paper.

\section{Author Contributions}

Conception and design: Ius, Sabatino. Acquisition of data: Ius, Somma, Altieri, Angileri, Certo, Cofano, Della Pepa, La Rocca, Panciani, Pignotti, Spena, Sabatino. Analysis and interpretation of data: Ius, Pignotti, Sabatino, Somma. Drafting the article: Ius, Somma, Pignotti, Sabatino. Critically revising the article: all authors. Reviewed submitted version of manuscript: Ius, Somma, Altieri, Angileri, Barbagallo, Cappabianca, Certo, Cofano, D’Elia, Della Pepa, Fontanella, Germanò, Garbossa, Isola, La Rocca, Maiuri, Olivi, Panciani, Pignotti, Skrap, Spena, Sabatino. Approved the final version of the manuscript on behalf of all authors: Ius. Statistical analysis: Ius, Isola. Administrative/ technical/material support: Ius, Pignotti. Study supervision: Ius, Angileri, Barbagallo, Cappabianca, Esposito, Fontanella, Germanò, Garbossa, Olivi, Skrap, Sabatino.

\section{Correspondence}

Tamara Ius: Santa Maria della Misericordia University Hospital, Udine, Italy.tamara.ius@gmail.com. 